\title{
HOMOLOGICAL PROPERTIES OF THE RING OF DIFFERENTIAL POLYNOMIALS ${ }^{1}$
}

\author{
BY JOHN H. COZZENS ${ }^{2}$
}

Communicated by Hyman Bass, July 27, 1969

The ring of differential polynomials over a universal differential field (Kolchin [7]), and the ring of twisted polynomials $\bar{F}_{2}[t, \rho]$, where $\bar{F}_{2}$ is an algebraic closure of $Z / 2 Z$ and $\rho$ is the automorphism of $\bar{F}_{2}$ defined by: $z \mapsto z^{2}$, "localized" at the multiplicative subset $\left\{t^{k} \mid k\right.$ an integer $\left.\geqq 0\right\}$, provide examples of a principal right and left ideal domain $R$, not a field, that is a right $V$-ring (i.e., each simple right $R$-module is injective). Such a ring was conjectured to exist by Carl Faith. Both examples are shown to have a unique simple right $R$-module. If $R$ is either example, then by definition of a right $V$-ring, every right $R$-module has a maximal submodule. Bass proved that if a ring $A$ satisfies the d.c.c. on principal left ideals, then $A$ has a bounded number of orthogonal idempotents and every right $A$ module has a maximal submodule. The above examples show that the converse is false, thus answering a question raised by Bass [1, p. 470].

The author is deeply indebted to his adviser, Professor Carl Faith, for suggesting the problems treated in this paper and for his generous advice and encouragement.

1. Differential polynomials and right $V$-rings. Throughout this paper each ring $R$ will be a ring with an identity element 1 , and each right $R$-module $M$ will be unitary in the sense that $x 1=x$ for all $x \in M$. Mod- $R$ will denote the category of all right $R$-modules.

Definition 1. A ring $R$ is a right $V$-ring (after Villamayor) in case the following equivalent conditions are satisfied:

AMS Subject Classifications. Primary 1615, 1625, 1646; Secondary 1280.

Key Words and Phrases. Differential polynomial ring, differential field, universal differential field, twisted polynomial ring, principal ideal domain, right V-ring, injective module, simple module, divisible module, left perfect ring.

1 This paper represents a portion of the author's $\mathrm{Ph} . \mathrm{D}$. thesis, written under the direction of Professor Carl Faith at Rutgers, The State University, while the author was a National Defense Education Act Fellow.

2 Present address: Columbia University, New York, New York 10027. 
(1) Each simple right $R$-module is injective.

(2) Each right ideal is the intersection of maximal right ideals.

(3) $\operatorname{Rad} M=0$ for all $M \in \operatorname{Mod}-R$.

1.1. Proposition (FAITH). If $R$ is a prime, right noetherian, right $V$-ring, then $R$ is simple.

For a proof of Proposition 1.1 and its generalizations see Faith $[3$, p. 130]. Further results on the structure of right $V$-rings may also be found in Faith [4].

Let $k$ be a field with derivation $D$ and let $k[y, D]$ denote the ring of differential polynomials in the indeterminate $y$ with coefficients in $k$, i.e., the additive group of $k[y, D]$ is the additive group of the ring of polynomials in the indeterminate $y$ with coefficients in $k$, and multiplication in $k[y, D]$ is defined by: $y a=a y+D(a)$ for all $a \in k$, and its consequences. Let $f=\sum_{i=1}^{n} a_{i} y^{i} \in k[y, D], a_{n} \neq 0$. Define the degree of $f, \delta(f)=n$. The following properties are immediate:

(1) $\delta(f g)=\delta(f)+\delta(g)$, for all $f, g \in k[y, D]$.

(2) For $f, g \in k[y, D]$, there exist $h, r \in k[y, D]$ such that $f=g h+r$ where $r=0$ or $\delta(r)<\delta(g)$ (a similar algorithm holds on the left).

Thus, by (2), $k[y, D]$ is a principal right and left ideal domain.

Let $k$ be a field of characteristic 0 and $D$ be a derivation of $k$. A result due to Kolchin asserts the existence of a field $U \supseteq k$ and a derivation $\bar{D}$ of $U$ extending $D$ such that the equation

$$
p\left(x, \bar{D}(x), \cdots, \bar{D}^{(n)}(x)\right)=0, \quad n \text { arbitrary, }
$$

has a solution $\xi \in U$ for all $p(X) \in U\left[X_{1}, \cdots, X_{n+1}\right]-U$. Furthermore, every homogeneous linear differential equation in $\bar{D}$ over $U$ has a nontrivial solution in $U$. Such a field $U$ is called a universal extension of $k$ or a universal differential field (Kolchin [7]).

Let $k$ be a universal differential field with derivation $D$. For the remainder of this section, we shall always denote $k[y, D]$ by $R$.

1.2. Lemma. Given $f=\sum_{i=1}^{n} a_{i} y^{i} \in R, a_{n}=1$, there exist $\alpha_{i} \in k$, $1 \leqq i \leqq n$, such that $f=\prod_{i=1}^{n}\left(y-\alpha_{i}\right)$.

Proof. By induction on $\delta(f)$. We shall determine $\alpha, b_{i} \in k, 2 \leqq i \leqq n$, such that

$$
f=\left(y^{n-1}+b_{2} y^{n-2}+\cdots+b_{n}\right)(y-\alpha) .
$$

By expanding equation (1), equating coefficients, and eliminating the $b_{i}$, an equation of the form

$$
p\left(x, D(x), \cdots, D^{(n)}(x)\right)=0
$$


results. By hypothesis, there exists an $\alpha \in k$ satisfying (2).

1.2 implies, in particular, that the only irreducible elements of $R$ are those of degree 1 . Hence, $V_{\alpha}=R /(y-\alpha) R$ is a simple right $R$ module for all $\alpha \in k$ and conversely.

1.3. Lemma. $V_{\alpha}=R /(y-\alpha) R$ is a divisible right $R$-module for all $\alpha \in k$.

Proof. By 1.2, it suffices to show that

$$
V_{\alpha}(y+\beta)=V_{\alpha} \quad \text { for all } \quad \alpha, \beta \in k .
$$

Equivalently, given $h \in R, \delta(h)=0$, there exist $f, g \in R$ such that

$$
f(y+\beta)+(y+\alpha) g=h .
$$

We shall determine $a, b \in k$ such that

$$
a(y+\beta)+(y+\alpha) b=h .
$$

Equation (2) is equivalent to an equation of the form

$$
D(b)+(\alpha-\beta) b=h .
$$

By hypothesis, there exists a $b \in k$ satisfying (3).

1.4 THEOREM. The ring $R$ has the following properties:

(a) $R$ is a principal right and left ideal domain.

(b) $R$ is simple.

(c) $R$ is a right $V$-ring.

(d) $R$ is not a field.

(e) $R$ has, up to isomorphism, a unique simple right $R$-module.

Proof. (a) is obvious by properties (1) and (2) of the ring $k[y, D]$. (b) is implied by 1.1 since $R$ is a domain.

(c) 1.3 , together with the fact that divisibility is equivalent to injectivity in a principal right ideal domain (Faith [4] or CartanEilenberg [2, p. 134]), implies that each simple right $R$-module is injective.

(d) Obvious.

(e) To show that $R /(y-\alpha) R \cong R /(y-\beta) R$ where $\alpha, \beta \in k$, we observe (see the proof of 1.3) that there exist nonzero $a, b \in k$ such that $a(y-\alpha)=(y-\beta) b$. The map

$$
R /(y-\alpha) R \rightarrow R /(y-\beta) R
$$

defined by

$$
r+(y-\alpha) R \mapsto a r+(y-\beta) R
$$

is the desired isomorphism. 
2. Twisted polynomials. Let $\bar{F}_{2}$ denote an algebraic closure of $Z / 2 Z$ and $\rho$, the automorphism of $\bar{F}_{2}$ defined by: $z \mapsto z^{2} . \bar{F}_{2}[t, \rho]$ will denote the ring of twisted polynomials in $t$ over $\bar{F}_{2}$ i.e., the additive group of $\bar{F}_{2}[t, \rho]$ is the additive group of the ring of polynomials in the indeterminate $t$ with coefficients in $\bar{F}_{2}$, and multiplication in $\bar{F}_{2}[t, \rho]$ is defined by: $t a=\rho(a) t$ for all $a \in \bar{F}_{2}$, and its consequences. It is well known that $\bar{F}_{2}[t, \rho]$ is a principal right and left ideal domain. Furthermore, it is easy to show that the only two-sided ideals of $\bar{F}_{2}[t, \rho]$ are those of the form $t^{k} \bar{F}_{2}[t, \rho], k$ an integer $\geqq 0$ (Jacobson [5]).

Let $R=\bar{F}_{2}[t, \rho], M=\left\{t^{k} \mid k\right.$ an integer $\left.\geqq 0\right\}$, and $R_{M}=\{a / m \mid a \in R$, $m \in M\}$. For $a / t^{k}, b / t^{k+i} \in R_{M}, a / t^{k}=b / t^{k+i}$ if and only if $b=t^{i} a$. We define addition and multiplication in $R_{M}$ as follows: $a / t^{k}+b / t^{k}$ $=(a+b) / t^{k}$ and $a / t^{i} b / t^{j}=\rho^{j}(a) b / t^{i+j}$ where $\rho^{j}(a)$ is that element of $R$ obtained by applying $\rho^{j}$ to all the coefficients of $a$ (Jacobson [6, p. 211]). Clearly, $R_{M}$ is a simple integral domain, not a field. Moreover, $R_{M}$ is a principal right and left ideal domain.

2.1. Lemma. Given $r=\sum_{i=1}^{n} a_{i} t^{i} \in R, a_{n}=1$, there exist $\alpha_{i} \in \bar{F}_{2}$, $1 \leqq i \leqq n$, such that $r=\prod_{i=1}^{n}\left(t-\alpha_{i}\right)$. In particular, the irreducible elements of $R$ are those of the form $t-\alpha, \alpha \in \bar{F}_{2}$.

Proof. Analogous to 1.2.

One readily sees that the simple right $R_{M}$-modules are of the form $R_{M} / p R_{M}$ where $p=t-\alpha, \alpha \neq 0 \in \bar{F}_{2}$.

2.2. Lemma. $R_{M} /(t-\alpha) R_{M}$ is a divisible right $R_{M}$-module for all $\alpha \in \bar{F}_{2}-\{0\}$.

Proof. Analogous to 1.3 .

2.3. THEOREM. The ring $R_{M}$ has the following properties:

(a) $R_{M}$ is a principal right and left ideal domain.

(b) $R_{M}$ is simple.

(c) $R_{M}$ is a right V-ring.

(d) $R_{M}$ is not a field.

(e) $R_{M}$ has, up to isomorphism, a unique simple right $R_{M}$-module.

Proof. Analogous to 1.4 .

\section{REFERENCES}

1. H. Bass, Finitistic dimension and a homological generalization of semi-primary rings, Trans. Amer. Math. Soc. 95 (1960), 466-488. MR 28 \#1212.

2. H. Cartan and S. Eilenberg, Homological algebra, Princeton Univ. Press, Princeton, N. J., 1956. MR 17, 1040. 
3. C. Faith, Lectures on injective modules and quotient rings, Lecture Notes in Math., no. 49, Springer-Verlag, Berlin, 1967. MR 37 \#2791. 1969.

4. - Algebra: Rings, modules and categories, Saunders, Philadelphia, Pa.,

5. N. Jacobson, The theory of rings, Amer. Math. Soc. Mathematical Surveys, vol. 2, Amer. Math. Soc., Providence, R.I., 1943. MR 5, 31.

6. - Structure of rings, rev. ed., Amer. Math. Soc. Colloq. Publ., vol. 37, Amer. Math. Soc., Providence, R. I., 1964. MR 36 \#5158.

7. E. R. Kolchin, Galois theory of differential fields, Amer. J. Math. 75 (1953), 753824. MR 15, 394; MR 15, 1140.

Rutgers University, New Brunswick, New Jersey 08903 